\title{
Design of a Low Index Silicon Micro Ring Resonators
}

\author{
Bobby Barua \\ Department of EEE, Ahsanullah University of Science and Technology, Dhaka, Bangladesh \\ Corresponding Author Email: bobby@aust.edu
}

Regular paper: Received: Apr. 29, 2020, Revised: Dec. 20, 2020, Accepted: Jan. 14, 2021, Available Online: Jan. 16, 2021, DOI: 10.29252/ijop.14.2.187

\begin{abstract}
Optical resonators where light will circulate in two opposite directions independently. Usually, the two distinct topologies of optical resonators are Linear and Ring. Two end mirrors with perpendicular light incidence have linear resonators (standing-wave resonators). In the other hand, there are no end mirrors in ring resonators; none of the resonator mirrors reflects light back into itself. Given its versatility and cost-effectiveness, it is beneficial to provide a low index ring resonator. Compared with existing high index technologies it can provide an attractive solution. The refractive index difference plays a key role in evaluating the properties of an optical dielectric waveguide, and a higher index contrast allows for shifting to greater integration scales and accessibility to new technologies and interfaces. However high contrast waveguides are harder to realize and more important, that their use does not raise any severe impediments. The intent of this article is to evaluate the impact of the waveguide features with regard to the correlation of the index and to investigate the complexities. This paper evaluates the influence of the index contrast on dielectric waveguide characteristics such as single mode system, losses, technical constraints, and available materials. Evaluation is developed by utilizing Rsoft CAD, known as BeamProp software, for ring resonators (single, double or triple optical loop). The main objective of this contribution is to achieve maximum flexibility and more productivity from the proposed design. This paper is an analogy for the design of optical waveguides, so that we can achieve flexibility by lowering the silicon index.
\end{abstract}

KEYWORDS: Low index waveguides, materials, micro-ring resonators, Rsoft CAD, Wireframe.

\section{I.INTRODUCTION}

In modern technology waveguides are essential components. They are used in the fiber optic cables that provide broadband internet, direct the light waves inside household electric appliances, and are often used in the complex technical equipment used in physical science and medicine [1].

Waveguides are not simply one kind of technology. In addition, there are several different wave-types that can be guided, e.g. optical, sonic or electromagnetic, and each type involves an entirely identical waveguide structure [2]. Without a waveguide, once emitted waves propagate in all directions. Additionally, waves without a waveguide are bound by the inverse square law. That implies that the intensity is inversely proportional to the distance covered as the waves move away from the source [3]. Since waveguides cause no loss of strength and as electromagnetic waves propagate so rapidly, they are incredibly rare communications technology tools [4].

A waveguide's geometry is an integral factor for it to carry out its intended role. An optical wave guide is a mechanism that directs a light wave to travel along a certain desired path [57] by constraining it. In recent decades, Silicon Photonics has received attention as an emerging technology in semiconductors for optical telecommunications and optical interconnections [8-10]. In recent years, [1113], Silicon Photonics has been one of the most promising platforms for photonic integration. This can be related mainly to the 
correlation to a very high index difference and the development of CMOS technology, which permits the use of facilities for processing electronics to build photonic circuits [14]. Passive silicone waveguide architectures seem to have an incredible reduction in the footprint of waveguides and particularly sensitive devices for wavelengths [15-16]. And a prime example of this is ring resonator. Ring resonators provide a significant function in the performance of silicon photonics, since silicon allows incredible small size ring resonators [17]. A typical ring resonator consists with an optical wave guide that is looped back on itself, so that a resonance occurs when the resonator's optical path length is exactly a whole sequence of wavelengths [18]. Therefore, ring-resonators allow different resonances. Single-mode strip waveguides (socalled photonic wires) may have bend radii around $5 \mu \mathrm{m}$ due to the very high refractive index contrast between silicon and its oxide (or air). This makes incredibly compact rings.

Moreover refractive index difference performs a critical role in determining the features of such an optical dielectric waveguide as well as a higher index difference allows for progressing towards wider integration scales and exposure to new technologies and functionalities[19-20]. Although high contrast waveguides are harder to realize and also more critical, but their use [21] does not raise any significant impediments.

The intent of such an analysis is to examine the influence of the waveguide characteristics with regard to the contrast of the index and to discuss the difficulties in designing of ring resonators. This paper explores the effect of the index contrast on dielectric waveguide characteristics along with single mode system, losses, fiber to waveguide coupling, technical constraints, and available materials. The investigation reveals that the sensitivity dependency on the polarization of directed modes in optical waveguide sensors is less in the low refractive index difference waveguide compared to the silica on silicon waveguides, but it gives the design engineers flexibility.

\section{Design Analysis}

We reproduce a particular material combination Core/ Cladding $(1.46 / 1.45 \mathrm{~nm})$ is considered to achieve a moderate refractive record distinction and the design reenactments are performed via BeamPROP The purpose of BeamPROP is to provide a specific propagation package for the production of light wave propagation in self-assertive wave geometry processing.

It helps the design engineers to describe the properties of a device's material and its functional geometry. BeamPROP estimates the optical field of the steady-state across a whole design database. Examination of this field in standard physical quantities like the optical power in a particular region of the circuit or the power traveling in a particular mode is also useful.

We have used the following settings for the above simulation:

Table 1. Parameters used for simulation

\begin{tabular}{|c|c|c|}
\hline Parameter & Value & Description \\
\hline Simulation & $\begin{array}{l}\text { Beam } \\
\text { PROP }\end{array}$ & $\begin{array}{l}\text { Sets BeamPROP as the } \\
\text { simulation engine to be } \\
\text { used }\end{array}$ \\
\hline $\begin{array}{l}\text { Waveguide } \\
\text { model } \\
\text { dimension }\end{array}$ & $2 \mathrm{D}$ & Creates 2D simulation \\
\hline $\begin{array}{l}\text { Free Space } \\
\text { Wavelength }\end{array}$ & 1.55 & $\begin{array}{l}\text { Sets the wavelength }(\mu \mathrm{m}) \\
\text { for the simulation }\end{array}$ \\
\hline $\begin{array}{l}\text { Background } \\
\text { Index }\end{array}$ & 1.45 & $\begin{array}{l}\text { Represents the real } \\
\text { refractive index of the } \\
\text { background material }\end{array}$ \\
\hline $\begin{array}{c}\text { Index } \\
\text { Difference }\end{array}$ & 0.01 & $\begin{array}{l}\text { Represents the difference } \\
\text { between a component and } \\
\text { the background material }\end{array}$ \\
\hline $\begin{array}{l}\text { Component } \\
\text { Width }\end{array}$ & 5 & $\begin{array}{l}\text { This sets the width of a } \\
\text { component }(\mu \mathrm{m})\end{array}$ \\
\hline
\end{tabular}

\section{A. Single Micro-Ring Resonator}

Figure 1 demonstrates a single-ring waveguide resonator configuration. An optical ring resonator is an array of waveguides of which at least one is a closed loop coupled with a certain light input and output. As light of the resonant wavelength is passes thru the loop from either the input waveguide, it develops up in intensity for several circular trips due to 
proactive interference and is throughput to the output loop waveguide which serves as a detector waveguide. As only a small few wavelengths are in the loop at resonance, the optical ring resonator performs as a filter. Two Y-branch waveguides are used for single ring resonator to couple with each other which forms a ring shape.

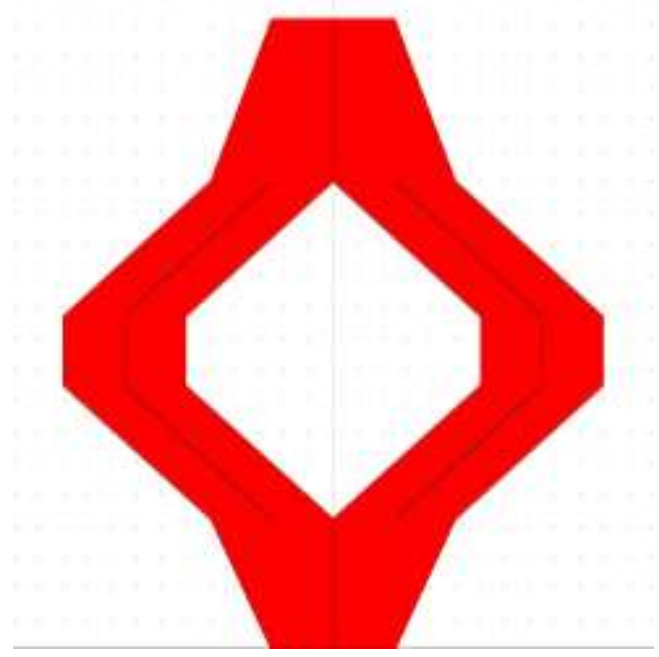

Fig. 1. Design of a single ring resonator waveguide.

\section{B. Multiple Ring Resonator Waveguide}

The two ring and three ring waveguide resonator configurations are shown in Figs. 2 and 3. Each ring resonator is coupled to each other in the systemically coupled configuration, and a signal to be extracted from the interface port to the drop port should travel across each resonator in series. With this serial power transmission, all resonators at a rising wavelength need to be precisely resonant. The resulting resonant path structure in the design of the system is physically determined by the separations between the ring resonators. In the perpendicular-coupled layout, all resonators of the waveguides are combined to with the input and drop port. Instead, the resonators are indirectly connected by the optical path lengths along the input and output waveguides which interconnect them. Those lengths decide the resonant line structures descriptions. An optical signal passes thru all ring resonators at the same time in parallel configuration. This softens the requirement where each ring should have exactly the same resonances. Instead, unaligned resonant frequencies contribute to several peaks, or line-shaped ripples. Additionally, as implied earlier, four or more ring waveguides can be coupled to each other to form an add/drop filter.

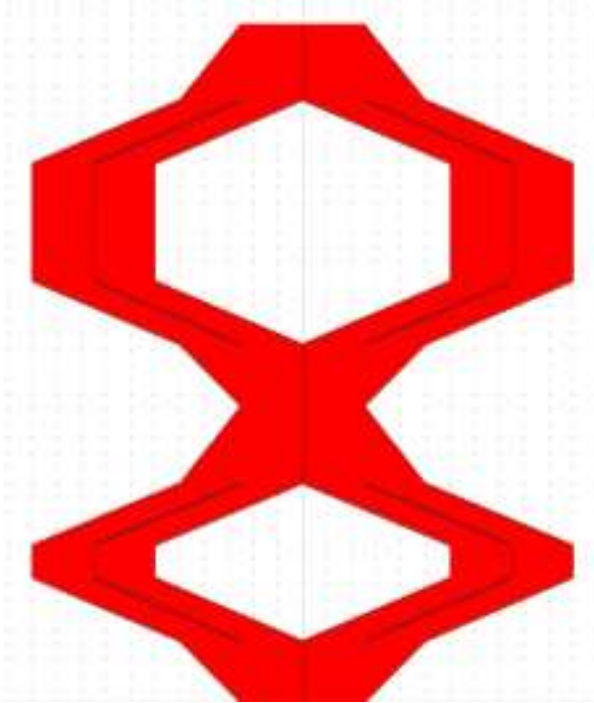

Fig. 2. Design of a double ring resonator waveguide.

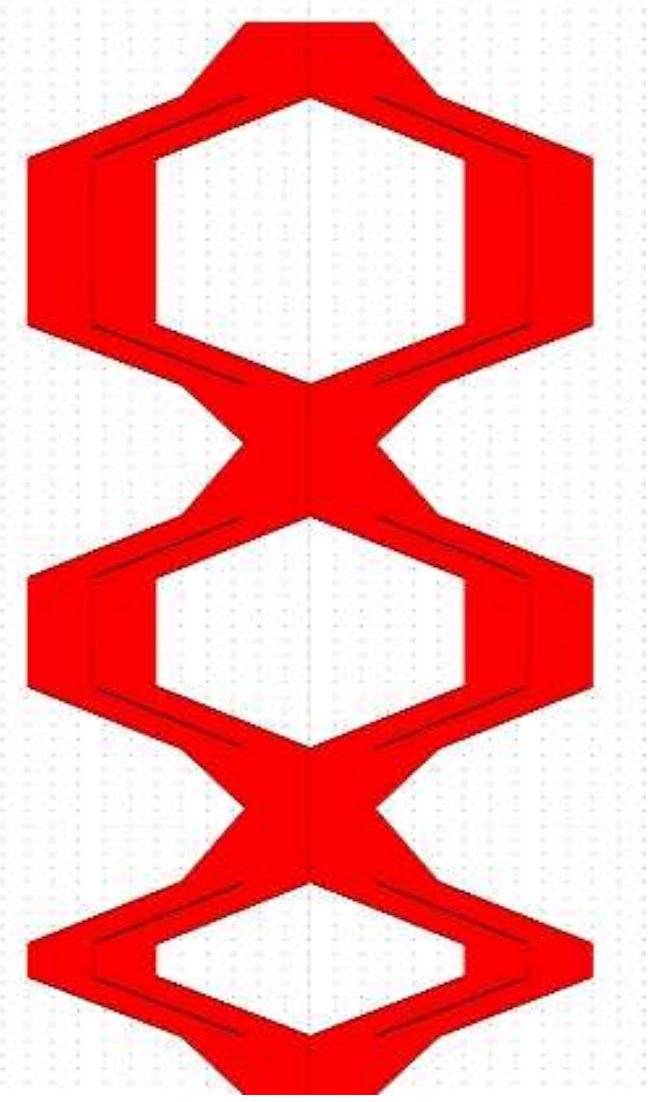

Fig. 3. Design of a Triple ring resonator waveguide. 


\section{III.EXTRAPOLATION OF SIMULATION RESULTS}

After the simulation, we can evaluate the characteristics of different Low Index Silicon Waveguides geometry. Tables also provide simulation parameters for different geometries. In 'Fiber' mode we conducted all of the simulation. Waveguide in fiber mode is considered the perfect waveguide for application.

Table 2 Parameters for simulation of micro-ring resonators

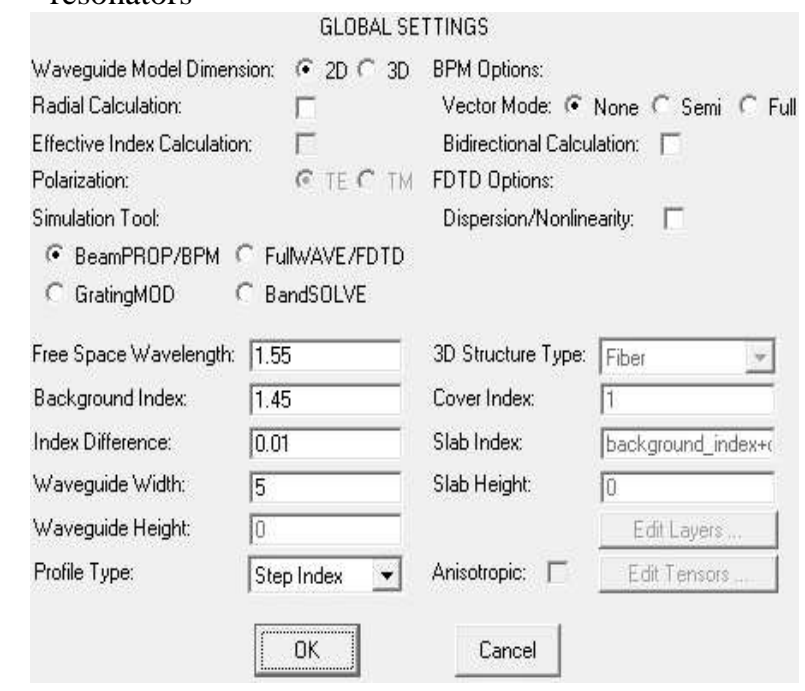
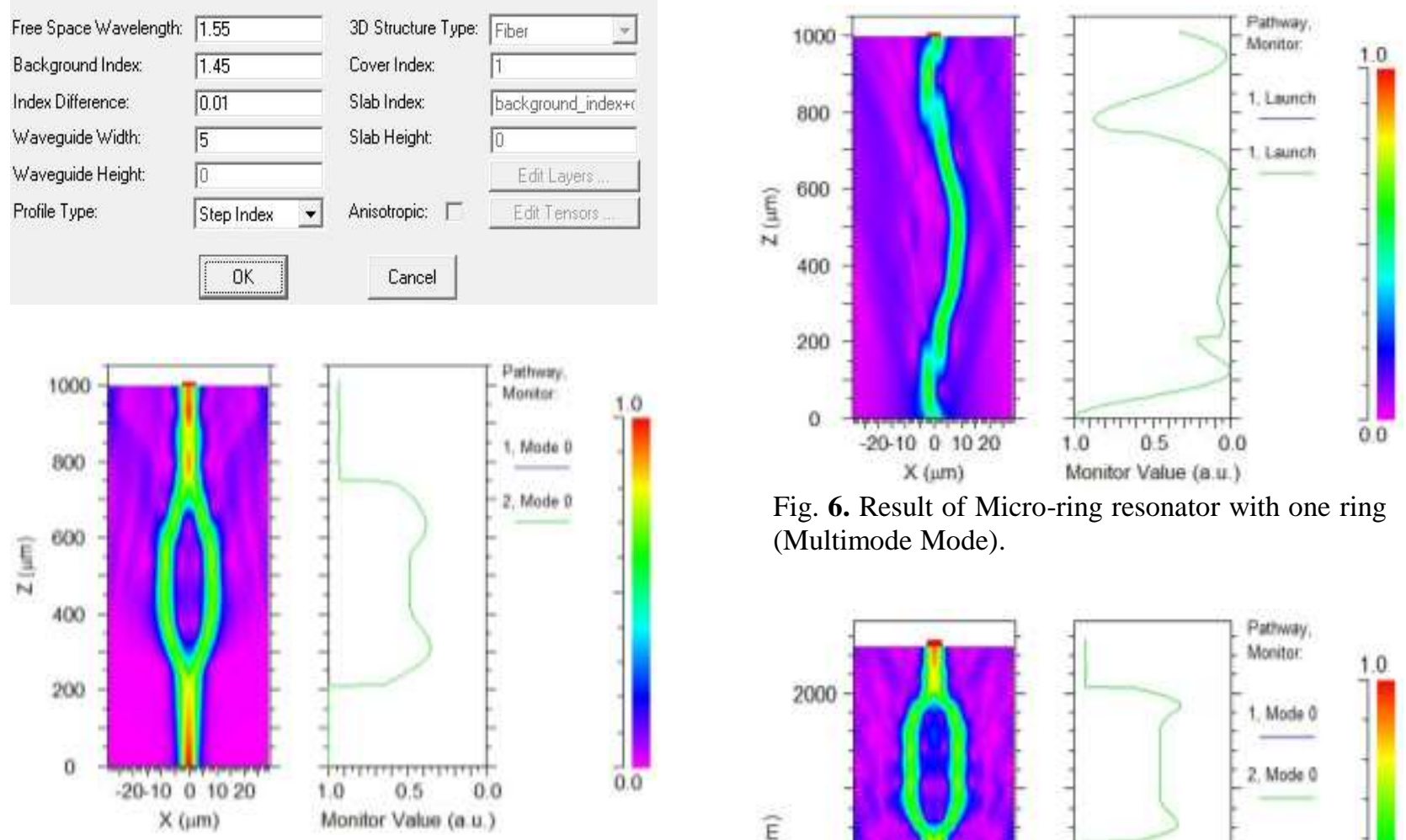

Fig. 6. Result of Micro-ring resonator with one ring (Multimode Mode).
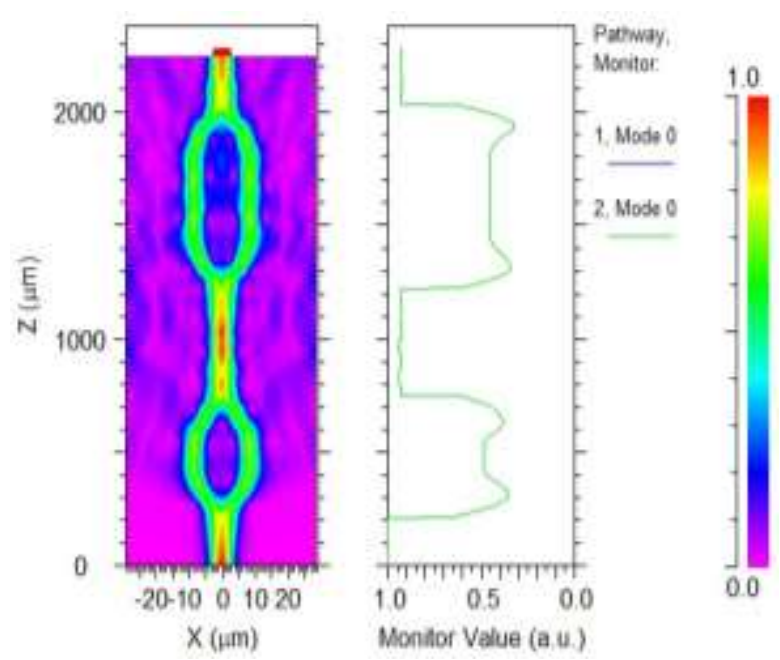

Fig. 7. Result of Micro-ring resonator with two rings (Slab Mode). 


\section{Comments on Simulation Results}

We conducted simulations for both Gaussian and multiple mode configurations for comparable geometries that are included in Figs. 5 and 6. It is noted from the simulation results that the power output is 85.5 percent in Gaussian mode and its input power is 33 percent in Multimode. So it is clear that the best solution overall is given by slab mode.

\section{Micro-Ring Resonator with Two Rings: Comments on simulation result}

Figure 7 reflects the micro-ring resonator simulation result with two loops. The double ring waveguide simulation is conducted with a very low difference in index. We found that the power output in slab mode is approximately 91 percent of the power input.

\section{* Micro-Ring Resonator with Three Rings:}

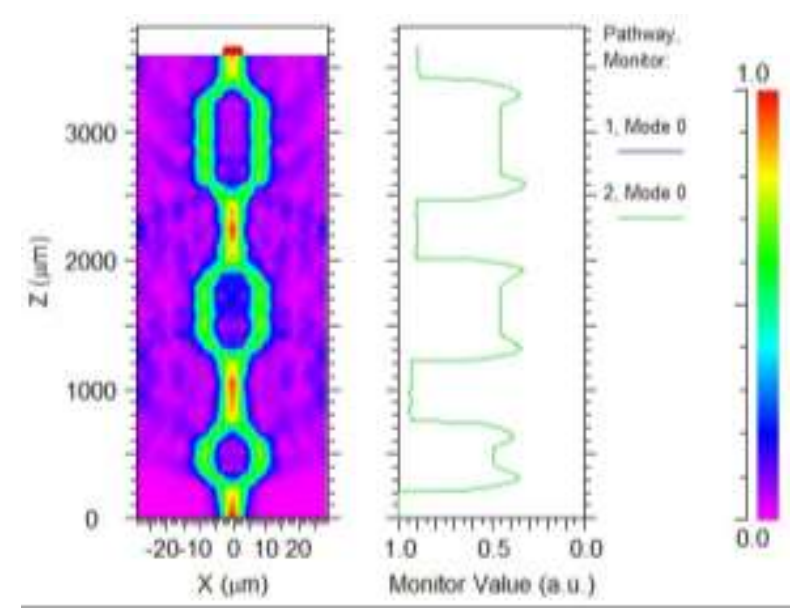

Fig. 8. Result of Micro-ring resonator with three rings (Slab Mode).

Figure 8 demonstrates the results of the simulation of micro-ring resonators using three loops. The consequence of the simulation shows that in a triple ring waveguide with a very slight index difference, the slab mode output power is almost 88 percent of its input power.

\section{IV.CONCLUSION}

The purpose of such a study is to investigate the effect of the waveguide characteristics on index contrast and to resolve the difficulties in the design of ring resonators. The investigation reveals that the susceptibility reliance on the polarization of directed modes in optical waveguide sensors is lower in the low refractive index gap waveguide than in silica on silicon waveguides, but it requires versatility for design engineers. We reproduce a specific material combination in order to achieve a rational refractive record differentiation, and design re-enactments are performed via BeamPROP. In 2D vision, all of the protests are carried out, which is why we just change the distance. We should have altered both the height and the width in 3D. The 3D slice view and Wireframe view illustrate the success of the architecture and it is noted that the reliability of the device from the integrated study of both figures is remarkable. The electronic transistors that will be replaced due to sluggish and decaying electron characteristics will be substituted by our designed photonic waveguides.

\section{REFERENCES}

[1] L. Pavesi and D.J. Lockwood, Topics in Applied Physics, Silicon Photonics, SpringerVerlag Berlin Heidelberg, 2004.

[2] D. Melati, A. Melloni, and F. Morichetti, "Real photonic waveguides: guiding light through imperfections," Adv. Opt. Photon. Vol. 6, pp. 156-224, 2014.

[3] D. Melati, F. Morichetti, and A. Melloni, "Modeling reflections induced by waveguide transitions," Opt. Quantum Electron. Vol. 35, pp. 1-8, 2012.

[4] M.J. Paniccia, "A perfect marriage: Optics and silicon," Optik \& Photonik, Vol. 2, pp. 34-38, 2011.

[5] J. Ahn, M. Fiorentino, R.G. Beausoleil, N. Binkert, A. Davis, D. Fattal, N.P. Jouppi, M. McLaren, C.M. Santori, R.S. Schreiber, S.M. Spillane, D. Vantrease, and Q. Xu "Devices and architectures for photonic chip-scale integration," Appl. Phys. A, Vol. 95, pp. 989997, 2009.

[6] D. Melati, A. Alippi, A. Annoni, N. Peserico, and A. Melloni, "Integrated all-optical MIMO demultiplexer for mode- and wavelengthdivision-multiplexed transmission," Optics Letters, Vol. 42, pp. 342-345, 2017.

[7] F. Morichetti, F. Toso, F, Zanetto, G. Ferrari, M. Sampietro, A. Melloni, and D.A.B. Miller, 
"Dynamically controlling optical beams with programmable silicon photonic meshes," Proceedings, Vol. 11283, Integrated Optics: Devices, Materials, and Technologies XXIV; 1128310, 2020.

[8] B. Jalali and S. Fathpour, "Silicon Photonics," J. Lightwave Technol. Vol. 24, pp. 4600 4615, 2006.

[9] J.C.C. Mak and J.K.S. Poon, "Multivariable Tuning Control of Photonic Integrated Circuits," IEEE J. Lightw. Technol. Vol. 35, pp. 1531-1541, 2017.

[10] M. Milanizadeh, S. Ahmadi, M. Petrini, D. Aguiar, R. Mazzanti, F. Zanetto, E. Guglielmi, M. Sampietro, F. Morichetti, and A. Melloni, "Control and calibration recipes for photonic integrated circuits," IEEE J. Sel. Top. Quantum Electron. Vol. 26, pp. 370-378, 2020.

[11]P. Dumais, D.J. Goodwill, D. Celo, J. Jiang, Ch. Zhang, F. Zhao, X. Tu, Ch. Zhang, Sh. Yan, J. He, M. Li, W. Liu, Y. Wei, D. Geng, H. Mehrvar, and E. Bernier "Silicon Photonic Switch Subsystem With 900 Monolithically Integrated Calibration Photodiodes and 64Fiber Package," IEEE J. Lightw. Technol. Vol. 36, pp. 233-238, 2018.

[12]F. Testa, C. J. Oton, Ch. Kopp, J.-M. Lee, R. Ortuño, R. Enne, S. Tondini, G. Chiaretti, A. Bianchi, P. Pintus, M.-S. Kim, D. Fowler, J. Á. Ayúcar, M. Hofbauer, M. Hofbauer, M. Mancinelli, M. Fournier, G. Battista Preve, N. Zecevic, C. L. Manganelli, C. Castellan, G. Parès, O. Lemonnier, F. Gambini, P. Labeye, M. Romagnoli, L. Pavesi, H. Zimmermann, F. D. Pasquale, and S. Stracca, "Design and Implementation of an Integrated Reconfigurable Silicon Photonics Switch Matrix in IRIS Project," IEEE J. Sel. Top. Quantum Electron, Vol. 22, pp. 155-168, 2016.

[13] G. Choo, S. Cai, B. Wang, C.K. Madsen, K. Entesari and S. Palermo, "Automatic MonitorBased Tuning of Reconfigurable Silicon Photonic APF-Based Pole/Zero Filters," IEEE J. Lightw. Technol. Vol. 36, pp. 1899-1911, 2018.

[14]P. Dong, "Silicon Photonic Integrated Circuits for Wavelength-Division Multiplexing Application," IEEE J. Sel. Top. Quantum Electron, Vol. 22, pp. 370-378, 2016.
[15] S. Grillanda, F. Morichetti, N. Peserico, P. Ciccarella, A. Annoni, M. Carminati, and A. Melloni, "Non-Invasive Monitoring of Mode Division Multiplexed Channels on a Silicon Photonic Chip," IEEE J. Lightw. Technol. Vol. 33, pp. 1197-1201, 2015.

[16] V.R. Almeida and M. Lipson, "Optical bistability on a silicon chip," Opt. Lett. Vol. 29, pp. 2387-2389, 2004.

[17]D. Aguiar, M. Milanizadeh, E. Guglielmi, F. Zanetto, G. Ferrari, M. Sampietro, F. Morichetti, and A. Melloni, "Automatic Tuning of Silicon Photonics Microring Filter Array for Hitless Reconfigurable AddDrop," J. Light. Technol. Vol. 73, pp. 3939 - 3947, 2019.

[18] H. Jayatilleka, H. Shoman, R. Boeck, N.A.F. Jaeger, L. Chrostowski, and S. Shekhar, "Automatic Configuration and Wavelength Locking of Coupled Silicon Ring Resonators," IEEE J. Lightw. Technol. Vol. 36, pp. 210$218,2018$.

[19]A. Melloni, R. Costa, G. Cusmai, and F. Morichetti, "The role of index contrast in dielectric optical waveguides," Int. J. Mater. Prod. Technol. Vol. 34, pp. 421-437, 2009.

[20]B. Barua, S.P. Majumder, and A. Melloni, "Characterization of Low index $\mathrm{Si}$ Waveguides," 19th International Conference on Computer and Information Technology (ICCIT), Vol. 95 , pp. 113-116, 2016.

[21]R. Soref, "Integrated-photonic switching structures," APL Photonics, Vol. 3, pp. 021101 (1-19), 2018.

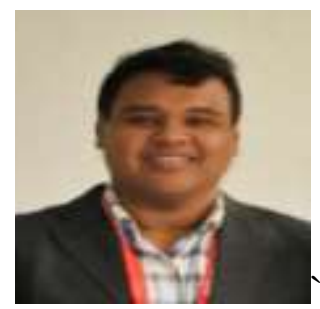

Dr. Bobby Barua was born at Dhaka, Bangladesh in December 1981. He is currently a Professor in the Department of Electrical and Electronic Engineering (EEE) at Ahsanullah University of Science \& Technology (AUST). $\mathrm{He}$ received his B.Sc. in Electrical \& Electronic Engineering (1st class with Honors) 
from AUST in 2003 and M.Sc. in Electrical \& Electronic Engineering (1st class Honors) from Bangladesh University of Engineering and Technology (BUET) in 2008. In 2018, he obtained his Ph.D. degree in Electrical \& Electronic Engineering from BUET in 2018.
He worked as research fellow at Politecnico Di Milano, Italy. His research interests include Free space Optical Communication Systems, Optical Fiber Communication Systems, Optical Networks, Soliton propagation, Satellite Communications, Mobile and Infrared communications with over 70 publication. 
THIS PAGE IS INTENTIONALLY LEFT BLANK. 